\title{
A mean-square bound for the lattice discrepancy of bodies of rotation with flat points on the boundary
}

\author{
by \\ Werner Georg NowaK (Vienna) \\ Dedicated to Professor Edmund Hlawka on his 90th birthday
}

1. Introduction. Let $\mathcal{B}$ denote a compact convex body in $s$-dimensional Euclidean space, $s \geq 2$, which contains the origin as an inner point and whose boundary $\partial \mathcal{B}$ is sufficiently smooth. The central question of the classical lattice point theory of large domains is to estimate the lattice point discrepancy of a linearly dilated copy $t \mathcal{B}$, i.e.,

$$
P_{\mathcal{B}}(t):=\#\left(t \mathcal{B} \cap \mathbb{Z}^{s}\right)-\operatorname{vol}(\mathcal{B}) t^{s},
$$

where $t$ is a large real parameter. For enlightening accounts on this topic, the reader is referred to E. Krätzel's monographs [14] and [15], to a recent survey article by A. Ivić, E. Krätzel, M. Kühleitner, and W. G. Nowak [10], and to M. Huxley's book [7] where he presented his breakthrough in the planar case ("discrete Hardy-Littlewood method").

If $\partial \mathcal{B}$ is of bounded nonzero Gaussian curvature throughout, the usual and plausible conjecture is that

$$
P_{\mathcal{B}}(t) \ll t^{\Theta_{s}+\varepsilon}
$$

for every $\varepsilon>0$, where $\Theta_{2}:=1 / 2$ and $\Theta_{s}:=s-2$ for $s \geq 3$. It is wellknown that for every dimension, $\Theta_{s}$ is the minimal possible value with this property, and that (1.2) is actually true for spheres of dimension $s \geq 4$, even with $\varepsilon=0$ if $s \geq 5$ : see, e.g., E. Krätzel [15, p. 227]. Quite recently, V. Bentkus and F. Götze [1] and F. Götze [3] established (1.2) for arbitrary ellipsoids of dimension $s \geq 5$.

However, for $s=2$ and 3, and for general bodies of higher dimensions, the proof or disproof of (1.2) remains an open problem. The sharpest known

2000 Mathematics Subject Classification: 11P21, 11K38, 52C07.

The author gratefully acknowledges support from the Austrian Science Fund (FWF) under project No. P18079-N12. 
estimates are due to W. Müller [24]. Researchers subsequently dealt with the task of verifying (1.2) "on average", i.e., showing that

$$
\int_{0}^{T}\left(P_{\mathcal{B}}(t)\right)^{2} d t \ll T^{2 \Theta_{s}+1+\varepsilon} .
$$

In fact, (1.3) was established for planar domains by D. G. Kendall [13] and the author [25], ultimately in the form of an asymptotics [26]. For dimensions $s \geq 4$, (1.3) was proved by W. Müller [23] who however had to leave open the case $s=3$. This gap was filled by A. Iosevich, E. Sawyer, and A. Seeger [8] who showed that

$$
\int_{0}^{T}\left(P_{\mathcal{B}}(t)\right)^{2} d t \ll \begin{cases}T^{2 \Theta_{s}+1} & \text { for } s \geq 4, \\ T^{3}(\log T)^{2} & \text { for } s=3 .\end{cases}
$$

The very last estimate comes rather close to the asymptotic formula known for the three-dimensional sphere $\mathcal{B}_{0}$, namely

$$
\int_{0}^{T}\left(P_{\mathcal{B}_{0}}(t)\right)^{2} d t=C T^{3} \log T+O\left(T^{3}(\log T)^{1 / 2}\right) .
$$

Cf. V. Jarník [12], and also Y.-K. Lau [22] who improved the error term to $O\left(T^{3}\right)$.

2. Recent developments and statement of the present result. The topic of this note will combine two recent trends in lattice point theory: On the one hand, increased interest arose in $\mathbb{R}^{3}$-bodies of rotation (with respect to one of the coordinate axes), denoted by $\mathcal{R}$ in what follows. For the case of nonzero curvature, F. Chamizo [2] obtained the upper bound

$$
P_{\mathcal{R}}(t) \ll t^{11 / 8+\varepsilon}
$$

while the papers by M. Kühleitner [20] and M. Kühleitner and W. G. Nowak [21] provided $\Omega$-results. A recent article of E. Krätzel and W. G. Nowak [19] gives a version of (2.1) with numerical constants, for the special case of an ellipsoid.

On the other hand, a number of papers investigated the influence of boundary points with curvature zero on the lattice discrepancy. While Krätzel's monograph [15] provides an enlightening survey of the planar case (which is comparatively well-understood), results for dimension 3 and higher can be found in the works of K. Haberland [4], E. Krätzel [16]-[18], and M. Peter [27]. These are all (pointwise) $O$-estimates, partially providing a precise evaluation of the contribution of an isolated flat point on $\partial \mathcal{B}$ to $P_{\mathcal{B}}(t)$, with a remainder of smaller order. 
In the present paper we shall take up both of these matters, in the aspect of a mean-square estimate in the sense of (1.3). We will consider an $\mathbb{R}^{3}$-body of rotation $\mathcal{R}$ (with respect to one of the coordinate axes), with smooth boundary $\partial \mathcal{R}$ of nonzero Gaussian curvature $\kappa$ throughout, except for the points of intersection of $\partial \mathcal{R}$ with the axis of rotation, where $\kappa$ may vanish. It will turn out that the contribution of these flat points to the lattice point discrepancy can be evaluated quite accurately, leaving a remainder term which is in mean-square "as small as it should be", in the sense of formula (1.3).

We remark parenthetically that if $\kappa$ vanished anywhere else on $\partial \mathcal{B}$, it would do so on a whole circle. This would presumably have a more dramatic effect on the lattice discrepancy. It seems much more difficult to obtain a sharp result in this general case.

Precise formulation of the present assumptions. Let $\varrho:[0, \pi] \rightarrow \mathbb{R}_{>0}$ be a function of class $C^{4}$, with $\varrho^{\prime}(0)=\varrho^{\prime}(\pi)=0$ and $\left(^{1}\right)$

$$
\varrho \varrho^{\prime \prime}-2 \varrho^{\prime 2}-\varrho^{2} \neq 0
$$

throughout $] 0, \pi[$. Suppose that $\varrho$ is analytic at $\pi$ and 0 . At these two values, the left-hand side of (2.2) may vanish, of orders (exactly) $N_{1}, N_{2} \geq 0$, as a function of $\theta$, the case that $\min \left(N_{1}, N_{2}\right)=0$ not being excluded. It will be convenient to write for short

$$
\alpha_{i}:=\frac{1}{N_{i}+2} \quad(i=1,2)
$$

Now

$$
\mathcal{C}=\{(x, y)=(\varrho(|\theta|) \cos \theta, \varrho(|\theta|) \sin \theta): \theta \in[-\pi, \pi]\}
$$

defines a smooth curve in the $(x, y)$-plane, symmetric with respect to the $x$-axis. Rotating $\mathcal{C}$ around the latter, we obtain a smooth surface in $(x, y, z)$ space, which we call $\partial \mathcal{R}$, where $\mathcal{R}$ is the compact convex body bounded by $\partial \mathcal{R}$. We denote by $a_{1}, a_{2}$ the minimal, resp., maximal $x$-coordinate on $\partial \mathcal{R}$. Obviously, the Gaussian curvature of $\partial \mathcal{R}$ vanishes at most at the points of intersection with the $x$-axis.

THEOREM. Suppose that the conditions stated above are satisfied, in particular, the Gaussian curvature of $\partial \mathcal{R}$ vanishes at most at the two points of intersection with the axis of rotation. Then for the number $A_{\mathcal{R}}(t)$ of lattice points in the linearly dilated body $t \mathcal{R}$ we have the asymptotic formula

$$
A_{\mathcal{R}}(t)=\operatorname{vol}(\mathcal{R}) t^{3}+\sum_{i=1}^{2} \sum_{j=2}^{N_{i}+1} d_{i, j}^{*} \lambda_{i, j}(t) t^{2-j \alpha_{i}}+\Delta_{\mathcal{R}}(t),
$$

$\left({ }^{1}\right)$ Recall that the curvature of a curve whose equation in polar coordinates is $\varrho=\varrho(\theta)$ is given, in absolute value, by $\left|\varrho(\theta) \varrho^{\prime \prime}(\theta)-2 \varrho^{\prime 2}(\theta)-\varrho^{2}(\theta)\right| /\left(\varrho^{2}(\theta)+\varrho^{\prime 2}(\theta)\right)^{3 / 2}$. 
where

$$
\begin{array}{r}
\lambda_{i, j}(t):=(2 \pi)^{-j \alpha_{i}} \Gamma\left(j \alpha_{i}\right) \sum_{k=1}^{\infty} k^{-1-j \alpha_{i}} \sin \left((-1)^{i} \pi j \alpha_{i} / 2-2 \pi k a_{i} t\right) \\
(i=1,2),
\end{array}
$$

and the remainder satisfies the mean-square estimate

$$
\int_{0}^{T}\left(\Delta_{\mathcal{R}}(t)\right)^{2} d t=O\left(T^{3+\varepsilon}\right)
$$

for each $\varepsilon>0$. The coefficients $d_{1, j}^{*}, d_{2, j}^{*}$ are computable, from the formulas (3.1)-(3.5) below. In particular, $d_{1,1}^{*}>0, d_{2,1}^{*}<0$.

Remarks. 1. It is easy to see that the error term satisfies in fact the pointwise upper estimate

$$
\Delta_{\mathcal{R}}(t)=O\left(t^{3 / 2+\varepsilon}\right)
$$

This is a straightforward consequence of the works of Krätzel [15]-[18], but also follows as a simple by-product from the argument in this paper: see the concluding remark at the end.

2. There is a crucial difference in the treatment of the problem, depending on whether there are boundary points of curvature zero or not. For $\kappa$ nonzero, the analysis leading to the results (1.3), (1.4) is based on the asymptotic expansion of the Fourier transform of the indicator function of the body $\mathcal{B}$, which is due to E. Hlawka [5], [6]. In the case that inf $\kappa=0$ the latter is not at our disposal. Thus we have to employ a quite different approach which uses a truncated Hardy identity (Lemma 1) and a transformation of trigonometric sums.

\section{Some auxiliary results}

Lemma 1. For integers $k \geq 0$, let as usual $r(k)$ denote the number of pairs $\left(m_{1}, m_{2}\right) \in \mathbb{Z}^{2}$ with $m_{1}^{2}+m_{2}^{2}=k$. Then for large real parameters $X, Y$, and any $\varepsilon>0$,

$$
\begin{aligned}
P(X):= & \sum_{0 \leq k \leq X} r(k)-\pi X \\
= & \frac{1}{\pi} X^{1 / 4} \sum_{1 \leq n \leq Y} \frac{r(n)}{n^{3 / 4}} \cos (2 \pi \sqrt{n X}-3 \pi / 4) \\
& +O\left(X^{1 / 2+\varepsilon} Y^{-1 / 2}\right)+O\left(Y^{\varepsilon}\right) .
\end{aligned}
$$

Proof. This is contained in formula (1.9) of A. Ivić [9]. 
Lemma 2. Let $F \in C^{4}[A, B], G \in C^{2}[A, B]$, and suppose that, for positive parameters $X, Y, Z$, we have $1 \leq B-A \ll X$ and

$$
\begin{array}{ll}
F^{(j)} \ll X^{2-j} Y^{-1} & \text { for } j=2,3,4, \quad\left|F^{\prime \prime}\right| \geq c_{0} Y^{-1}, \\
G^{(j)} \ll X^{-j} Z & \text { for } j=0,1,2,
\end{array}
$$

throughout the interval $[A, B]$, with some constant $c_{0}>0$. Let $\mathcal{J}^{\prime}$ denote the image of $[A, B]$ under $F^{\prime}$, and $F^{*}$ the inverse function of $F^{\prime}$. Then

$$
\begin{aligned}
\sum_{A<m \leq B} G(m) e(F(m)) \\
=e\left(\frac{\operatorname{sgn}\left(F^{\prime \prime}\right)}{8}\right) \sum_{k \in \mathcal{J}^{\prime}} \frac{G\left(F^{*}(k)\right)}{\sqrt{\left|F^{\prime \prime}\left(F^{*}(k)\right)\right|}} e\left(F\left(F^{*}(k)\right)-k F^{*}(k)\right) \\
\quad+O\left(Z\left(\sqrt{Y}+\log \left(2+\operatorname{length}\left(\mathcal{J}^{\prime}\right)\right)\right)\right) .
\end{aligned}
$$

Proof. Transformation formulas of this kind are quite common, though often with worse error terms. This very sharp version can be found as Theorem 8.16, together with formula (8.47), in the recent monograph [11] of H. Iwaniec and E. Kowalski.

For our argument it will be essential to have at hand a close analysis of the situation near the points where the Gaussian curvature (possibly) vanishes. To this end, let $\mathcal{C}_{+}$denote the upper half of $\mathcal{C}$, and set $\mathcal{C}_{+}=\{(\varrho(\theta) \cos \theta, \varrho(\theta) \sin \theta): \theta \in[0, \pi]\}=\left\{(x, y): a_{1} \leq x \leq a_{2}, y=f(x)\right\}$, with $a_{1}:=-\varrho(\pi)<0<a_{2}:=\varrho(0)$. This defines $f:\left[a_{1}, a_{2}\right] \rightarrow \mathbb{R}_{>0}$ as a strictly positive $C^{4}$-function on $] a_{1}, a_{2}\left[\right.$, with $f\left(a_{1}\right)=f\left(a_{2}\right)=0$, and $f^{\prime \prime}$ strictly negative throughout. By our assumptions, for each of the $a_{i}$ 's, and $(x, y) \in \mathcal{C}_{+}$in a suitable neighborhood of $\left(a_{i}, 0\right)$,

$$
x=a_{i}+c_{i} y^{N_{i}+2}+\sum_{m=1}^{\infty} c_{i, m} y^{N_{i}+2+m} \quad\left(c_{i} \neq 0\right) .
$$

Consequently,

$$
y=f(x)=\sum_{j=1}^{\infty} d_{i, j}\left|x-a_{i}\right|^{\alpha_{i} j}, \quad d_{i, 1}=\left|c_{i}\right|^{-\alpha_{i}} \neq 0,
$$

and the other $d_{i, j}$ 's can be computed recursively from the $c_{i, m}$ 's. It thus follows that, for $r=0,1,2, \ldots$,

$$
f^{(r)}(x) \asymp\left|x-a_{i}\right|^{\alpha_{i}-r}
$$

for $x$ close to $a_{i}$. Similarly, we deduce that

$$
\frac{d^{r}}{d x^{r}}(\sqrt{f(x)}) \asymp\left|x-a_{i}\right|^{\alpha_{i} / 2-r}
$$


for $r=0,1,2, \ldots$, and $x$ near $a_{i}$. Furthermore,

$$
\frac{d}{d x}\left(f^{2}(x)\right)=\sum_{j=2}^{\infty} d_{i, j}^{*}\left|x-a_{i}\right|^{\alpha_{i} j-1},
$$

with $d_{i, 2}^{*}=2 \alpha_{i} d_{i, 1}^{2}(-1)^{i+1}$, again in an appropriate neighborhood of $a_{i}$.

For our proof we will also need some knowledge about the tac-function of $\mathcal{R}$,

$$
H(u, v, w):=\max _{(x, y, z) \in \mathcal{R}}(u x+v y+w z)
$$

and the polar body $\mathcal{R}^{*}$, given by $H(u, v, w) \leq 1$. The connection between the smoothness and curvature of $\partial \mathcal{R}$ and of $\partial \mathcal{R}^{*}$ has been neatly worked out in W. Müller [23, Lemma 1]. It is clear that $H$ and thus $\mathcal{R}^{*}$ is again invariant under rotations about the first coordinate axis. Let $\mathcal{C}_{+}^{*}$ denote the intersection of $\partial \mathcal{R}^{*}$ with the closed upper half of the $(u, v)$-plane, i.e., $\mathcal{C}_{+}^{*}$ is the polar curve of $\mathcal{C}_{+}$. Then

$$
\mathcal{C}_{+}^{*}=\left\{(u, v): 1 / a_{1} \leq u \leq 1 / a_{2}, v=h(u)\right\},
$$

where $h:\left[1 / a_{1}, 1 / a_{2}\right] \rightarrow \mathbb{R}_{\geq 0}$ is a strictly positive $C^{3}$-function on $] 1 / a_{1}, 1 / a_{2}[$, with $h\left(1 / a_{1}\right)=h\left(1 / a_{2}\right)=0$ (cf. W. Müller [23, Lemma 1]).

LEMMA 3. With the conditions and definitions stated,

$$
\sup _{1 / a_{1}<u<1 / a_{2}}\left|h(u) h^{\prime}(u)\right|<\infty .
$$

Further, as $u \rightarrow 1 / a_{i}(i=1,2),\left|(d / d u)\left(h(u) h^{\prime}(u)\right)\right|$ either is bounded or tends to $\infty$.

Proof. Let the real numbers $x \in] a_{1}, a_{2}[$ and $u \in] 1 / a_{1}, 1 / a_{2}[$ be such that the points $(x, f(x))$ and $(u, h(u))$ are polar reciprocal to each other, i.e.,

$$
1=x u+f(x) h(u)=\max _{(\xi, \eta) \in \mathcal{C}_{+}}(\xi u+\eta h(u))=\max _{(\mu, \nu) \in \mathcal{C}_{+}^{*}}(x \mu+f(x) \nu) .
$$

Plainly, $(x, f(x)) \rightarrow\left(a_{i}, 0\right)$ if $(u, h(u)) \rightarrow\left(1 / a_{i}, 0\right)$, and vice versa. We may assume that $u$ is close to $1 / a_{i}(i=1$ or 2$)$, since elsewhere the boundedness of $h h^{\prime}$ is obvious. Then $x$ belongs to a suitable neighborhood of $a_{i}$, and the inverse function $x=x(y)$ is well-defined and given in an explicit form by (3.1). By elementary calculus, for $y=f(x) \Leftrightarrow x=x(y)$, we have

$$
0=u+f^{\prime}(x) h(u)=x^{\prime}(y) u+h(u)=x+f(x) h^{\prime}(u)=x(y)+y h^{\prime}(u) .
$$

Consequently, $h(u)=-x^{\prime}(y) u$ and $h^{\prime}(u)=-x(y) / y$, thus

$$
h(u) h^{\prime}(u)=u x(y) x^{\prime}(y) / y \ll 1,
$$

since $x^{\prime}(y) / y$ remains bounded as $y \rightarrow 0$, in view of (3.1). This verifies the first part of Lemma 3. 
Moreover, eliminating $h(u)$ from the pair of equations

$$
1=x(y) u+y h(u), \quad 0=x^{\prime}(y) u+h(u),
$$

we see that $u=\left(x(y)-x^{\prime}(y) y\right)^{-1}$, hence

$$
\frac{d u}{d y}=\frac{x^{\prime \prime}(y) y}{\left(x(y)-x^{\prime}(y) y\right)^{2}} \text {. }
$$

Therefore, using again (3.7), we obtain

$$
\begin{aligned}
\frac{d}{d u}\left(h(u) h^{\prime}(u)\right) & =\frac{d}{d y}\left(\frac{x x^{\prime}}{x y-x^{\prime} y^{2}}\right)\left(\frac{d u}{d y}\right)^{-1} \\
& =\frac{-x^{2} x^{\prime}+2 x x^{\prime 2} y-x^{\prime 3} y^{2}+x^{2} x^{\prime \prime} y}{x^{\prime \prime} y^{3}},
\end{aligned}
$$

where we have suppressed the argument $y$ of $x, x^{\prime}, x^{\prime \prime}$ for short. By (3.1), for small $y$ this is equal to a Laurent series, resp., Taylor series in $y$.

Lemma 4. For a large real parameter $X$ and the tac-function $H$ defined above, we have the asymptotics

$$
N(X):=\#\left\{(m, n) \in \mathbb{Z} \times \mathbb{Z}_{\geq 0}: H(m, \sqrt{n}, 0) \leq X\right\}=C X^{3}+O(X)
$$

with a constant $C>0$. Furthermore, for $0<\delta<1$,

$$
\begin{aligned}
N_{\delta}(X) & :=\#\left\{(m, n) \in \mathbb{Z} \times \mathbb{Z}_{\geq 0}: H(m, \sqrt{n}, 0) \leq X, \sqrt{n} \leq \delta|m|\right\} \\
& =C_{\delta} X^{3}+O(X),
\end{aligned}
$$

with a positive $C_{\delta} \ll \delta^{2}$ and the $O$-constant independent of $\delta$. As a consequence, for large $X$ and $0<\omega, \delta<1$,

$$
\begin{aligned}
& \#\left\{(m, n) \in \mathbb{Z} \times \mathbb{Z}_{\geq 0}:|H(m, \sqrt{n}, 0)-X|<\omega\right\} \ll X^{2} \omega+X, \\
& \#\left\{(m, n) \in \mathbb{Z} \times \mathbb{Z}_{\geq 0}:|H(m, \sqrt{n}, 0)-X|<\omega, \sqrt{n} \leq \delta|m|\right\} \\
& \\
& \ll X^{2} \omega \delta^{2}+X .
\end{aligned}
$$

Proof. Let $\mathcal{D}_{+}^{*}$ denote the compact planar domain bounded by the curve $\mathcal{C}_{+}^{*}$ and the $u$-axis. Obviously,

$$
\begin{aligned}
N(X) & =\#\left\{(m, n) \in \mathbb{Z} \times \mathbb{Z}_{\geq 0}:(m, \sqrt{n}) \in X \mathcal{D}_{+}^{*}\right\} \\
& =\sum_{\left(1 / a_{1}\right) X \leq m \leq\left(1 / a_{2}\right) X}\left(1+\left[X^{2} h^{2}(m / X)\right]\right) \\
& =X^{2} \sum_{\left(1 / a_{1}\right) X \leq m \leq\left(1 / a_{2}\right) X} h^{2}(m / X)+O(X) \\
& =X^{2} \int_{\left(1 / a_{1}\right) X}^{\left(1 / a_{2}\right) X} h^{2}(u / X) d u+2 X \int_{\left(1 / a_{1}\right) X}^{\left(1 / a_{2}\right) X} \psi(u) h(u / X) h^{\prime}(u / X) d u+O(X),
\end{aligned}
$$


by the Euler-MacLaurin formula (see E. Krätzel [14, p. 20]), with $\psi(u):=$ $u-[u]-1 / 2$. Here the first integral equals $C X$ with $C=\int_{1 / a_{1}}^{1 / a_{2}} h^{2}(\xi) d \xi$, which yields the main term of (3.9). Further, for any interval $\left.\left[\beta_{1}, \beta_{2}\right] \subset\right] 1 / a_{1}, 1 / a_{2}[$, an integration by parts gives

$$
\begin{aligned}
2 X \int_{\beta_{1} X}^{\beta_{2} X} \psi(u) h(u / X) h^{\prime}(u / X) d u & \\
= & 2 X\left(h\left(\beta_{2}\right) h^{\prime}\left(\beta_{2}\right) \psi_{1}\left(\beta_{2} X\right)-h\left(\beta_{1}\right) h^{\prime}\left(\beta_{1}\right) \psi_{1}\left(\beta_{1} X\right)\right) \\
& -2 \int_{\beta_{1} X}^{\beta_{2} X}\left(h(u / X) h^{\prime \prime}(u / X)+h^{\prime 2}(u / X)\right) \psi_{1}(u) d u
\end{aligned}
$$

where

$$
\psi_{1}(u):=\int_{0}^{u} \psi(v) d v \ll 1 .
$$

If $h h^{\prime \prime}+h^{\prime 2}=\left(h h^{\prime}\right)^{\prime}$ is bounded on $] 1 / a_{1}, 1 / a_{2}$ [, we simply let $\beta_{1} \rightarrow 1 / a_{1}$ and $\beta_{2} \rightarrow 1 / a_{2}$ to obtain the desired bound $O(X)$ for the remainder. In case $\left(h h^{\prime}\right)^{\prime}$ is unbounded near $1 / a_{1}$ (say), we choose $\beta_{1}>1 / a_{1}$ such that $\left(h h^{\prime}\right)^{\prime}$ has no sign change on $\left.] 1 / a_{1}, \beta_{1}\right]$. By the second mean-value theorem and Lemma 3,

$$
\int_{\left(1 / a_{1}\right) X}^{\beta_{1} X} \psi(u) h(u / X) h^{\prime}(u / X) d u \ll \sup _{] 1 / a_{1}, \beta_{1}\right]}\left|h h^{\prime}\right| \ll 1 .
$$

A similar reasoning holds near $1 / a_{2}$ if necessary. On the remaining interval $\left[\beta_{1} X, \beta_{2} X\right],(3.12)$ readily yields the bound $O(X)$ and thus completes the proof of (3.9). Quite similarly,

$$
\begin{aligned}
N_{\delta}(X)= & \sum_{\left(1 / a_{1}\right) X \leq m \leq\left(1 / a_{2}\right) X} \min \left(X^{2} h^{2}(m / X), \delta^{2} m^{2}\right)+O(X) \\
= & \int_{\left(1 / a_{1}\right) X}^{\left(1 / a_{2}\right) X} \min \left(X^{2} h^{2}(u / X), \delta^{2} u^{2}\right) d u \\
& +\int_{\left(1 / a_{1}\right) X}^{\left(1 / a_{2}\right) X} \psi(u) \frac{d}{d u}\left(\min \left(X^{2} h^{2}(u / X), \delta^{2} u^{2}\right)\right) d u+O(X) .
\end{aligned}
$$

Here it is important to notice that the equation $X^{2} h^{2}(u / X)=\delta^{2} u^{2}$ can have at most two solutions in $] X / a_{1}, X / a_{2}$ [. To see this, the change of variable $u / X \mapsto u$ gives $h(u)= \pm \delta u, 1 / a_{1}<u<1 / a_{2}$. Recalling the first equation of (3.6) leads to $f^{\prime}(x)=\mp 1 / \delta$, with $a_{1}<x<a_{2}$. By convexity of the arc $\mathcal{C}_{+}$, this has at most two solutions. 
The first integral in (3.13) equals $C_{\delta} X^{3}$ with

$$
C_{\delta}:=\int_{1 / a_{1}}^{1 / a_{2}} \min \left(h^{2}(\xi), \delta^{2} \xi^{2}\right) d \xi \ll \delta^{2} .
$$

The remainder integral can be treated as before, with the bound $O(X)$, since for any interval $I \subseteq] X / a_{1}, X / a_{2}[$,

$$
\int_{I} \psi(u) \delta^{2} u d u \ll X
$$

The deduction of (3.11) from (3.9), (3.10) is trivial.

Lemma 5. For an arbitrary finite index set $\mathcal{M}$, let $\left(\gamma_{m}\right)_{m \in \mathcal{M}}$ be a complex sequence and let $\left(\xi_{m}\right)_{m \in \mathcal{M}}$ be reals. Then, for any real $T_{0}$ and $T>0$,

$$
\int_{T_{0}}^{T_{0}+T}\left|\sum_{m \in \mathcal{M}} \gamma_{m} e\left(\xi_{m} t\right)\right|^{2} d t \leq 3 T \sum_{\substack{m_{1}, m_{2} \in \mathcal{M} \\\left|\xi_{m_{1}}-\xi_{m_{2}}\right|<1 / T}}\left|\gamma_{m_{1}} \gamma_{m_{2}}\right|
$$

Proof. This is essentially Lemma 7.1 in H. Iwaniec and E. Kowalski [11].

4. Asymptotic evaluation of the main terms. For a large parameter $t$ it follows, with the definitions of Section 3, that

$$
\begin{aligned}
A_{\mathcal{R}}(t) & =\sum_{a_{1} t \leq m \leq a_{2} t}\left(\sum_{0 \leq k \leq t^{2} f^{2}(m / t)} r(k)\right) \\
& =\pi t^{2} \sum_{a_{1} t \leq m \leq a_{2} t} f^{2}(m / t)+\sum_{a_{1} t \leq m \leq a_{2} t} P\left(t^{2} f^{2}(m / t)\right) .
\end{aligned}
$$

We proceed to evaluate the first sum on the right-hand side, postponing the mean-square estimation of the last one to the next section. By the EulerMacLaurin formula,

$$
\begin{aligned}
\pi t^{2} \sum_{a_{1} t \leq m \leq a_{2} t} f^{2}(m / t) & \\
& =\pi t^{2} \int_{a_{1} t}^{a_{2} t} f^{2}(\tau / t) d \tau+\pi t^{2} \int_{a_{1} t}^{a_{2} t} \psi(\tau) \frac{d}{d \tau}\left(f^{2}(\tau / t)\right) d \tau \\
& =\operatorname{vol}(\mathcal{R}) t^{3}+\pi t^{2} \int_{a_{1}}^{a_{2}} \psi(t x) \frac{d}{d x}\left(f^{2}(x)\right) d x
\end{aligned}
$$

By (3.5),

$$
\frac{d}{d x}\left(f^{2}(x)\right)=\sum_{j=2}^{2 N_{1}+3} d_{1, j}^{*}\left(x-a_{1}\right)^{\alpha_{1} j-1}+\sum_{j=2}^{2 N_{2}+3} d_{2, j}^{*}\left(a_{2}-x\right)^{\alpha_{2} j-1}+\Phi(x),
$$


with $\Phi \in C^{1}\left[a_{1}, a_{2}\right]$. Integrating by parts and using again $\psi_{1}(u) \ll 1$, we obtain

$$
t^{2} \int_{a_{1}}^{a_{2}} \psi(t x) \Phi(x) d x=O(t)
$$

The same argument works for $\left|x-a_{i}\right|^{\alpha_{i} j-1}$ instead of $\Phi(x)$, with $N_{i}+2 \leq$ $j \leq 2 N_{i}+3, i \in\{1,2\}$. In the subsequent analysis we may thus replace the upper summation limits in the sums from (4.3) by $N_{1}+1$, resp., $N_{2}+1$. To deal with the first of these remaining sums, we use the Fourier series

$$
\psi(z)=-\frac{1}{\pi} \sum_{k=1}^{\infty} \frac{1}{k} \sin (2 \pi k z) \quad(z \notin \mathbb{Z})
$$

and an obvious shift of variable. For $j=2, \ldots, N_{1}+1$, we conclude that

$$
\int_{a_{1}}^{a_{2}} \psi(t x)\left(x-a_{1}\right)^{\alpha_{1} j-1} d x=-\frac{1}{\pi} \sum_{k=1}^{\infty} \frac{1}{k} \int_{0}^{a_{2}-a_{1}} x^{\alpha_{1} j-1} \sin \left(2 \pi k t\left(a_{1}+x\right)\right) d x .
$$

An integration by parts shows that

$$
\int_{a_{2}-a_{1}}^{\infty} x^{\alpha_{1} j-1} \sin \left(2 \pi k t\left(a_{1}+x\right)\right) d x=O\left((k t)^{-1}\right) .
$$

Further,

$$
\begin{aligned}
\int_{0}^{\infty} x^{\alpha_{1} j-1} \sin \left(2 \pi k t\left(a_{1}+x\right)\right) d x & =\Im\left(e\left(a_{1} k t\right)(k t)^{-\alpha_{1} j} \int_{0}^{\infty} \tau^{\alpha_{1} j-1} e(\tau) d \tau\right) \\
& =\Im\left(e\left(a_{1} k t\right)(2 \pi k t)^{-\alpha_{1} j} \Gamma\left(\alpha_{1} j\right) e\left(\alpha_{1} j / 4\right)\right) \\
& =\Gamma\left(\alpha_{1} j\right)(2 \pi k t)^{-\alpha_{1} j} \sin \left(2 \pi a_{1} k t+\pi \alpha_{1} j / 2\right),
\end{aligned}
$$

using well-known formulas for the last integral (cf., e.g., H. Rademacher $[28$, p. 82]). Collecting the results, we get

$$
\pi t^{2} \int_{a_{1}}^{a_{2}} \psi(t x)\left(\sum_{j=2}^{N_{1}+1} d_{1, j}^{*}\left(x-a_{1}\right)^{\alpha_{1} j-1}\right) d x=\sum_{j=2}^{N_{1}+1} d_{1, j}^{*} \lambda_{1, j}(t) t^{2-j \alpha_{1}}+O(t)
$$

where $\lambda_{i, j}(t)$ has been defined in our Theorem. Dealing quite similarly with the second sum coming from (4.3), and recalling (4.2), (4.4), we obtain altogether

$$
\pi t^{2} \sum_{a_{1} t \leq m \leq a_{2} t} f^{2}(m / t)=\operatorname{vol}(\mathcal{R}) t^{3}+\sum_{i=1}^{2} \sum_{j=2}^{N_{i}+1} d_{i, j}^{*} \lambda_{i, j}(t) t^{2-j \alpha_{i}}+O(t) .
$$


5. Estimating the remainder in mean-square. It remains to deal with the last sum in (4.1), i.e., to show that

$$
\int_{T}^{2 T}\left(\sum_{a_{1} t \leq m \leq a_{2} t} P\left(t^{2} f^{2}(m / t)\right)\right)^{2} d t \ll T^{3+\varepsilon} .
$$

For given large $T$ we divide $\left.] a_{1},\left(a_{1}+a_{2}\right) / 2\right]$ and $\left.]\left(a_{1}+a_{2}\right) / 2, a_{2}\right]$ into dyadic subintervals $\left.\left.\left.\left.\mathcal{J}^{(1, r)}=\right] u^{(1, r+1)}, u^{(1, r)}\right], \mathcal{J}^{(2, r)}=\right] u^{(2, r)}, u^{(2, r+1)}\right], 0 \leq r \leq R$, where $u^{(i, r)}:=a_{i}-(-1)^{i} 2^{-r-1}\left(a_{2}-a_{1}\right)$, and $R$ is chosen such that the shortest of these intervals are of length $\asymp T^{-1}$. Dropping the superscripts for short, we write $\mathcal{J}$ for any of these subintervals, whose number is obviously $O(\log T)$. Let $\mathcal{K}:=\left[a_{1}, a_{2}\right] \backslash \bigcup \mathcal{J}$; then $|\mathcal{K}| \asymp T^{-1}$, and the trivial bound $P(z) \ll \sqrt{z}$ readily implies

$$
\int_{T}^{2 T}\left(\sum_{m \in t \mathcal{K}} P\left(t^{2} f^{2}(m / t)\right)\right)^{2} d t \ll T^{3} .
$$

Thus it suffices to prove that, for each $\mathcal{J}$ and $\varepsilon>0$,

$$
\int_{T}^{2 T}\left(\sum_{m \in t \mathcal{J}} P\left(t^{2} f^{2}(m / t)\right)\right)^{2} d t \ll T^{3+\varepsilon} .
$$

For every $t \in[T, 2 T]$ and $m \in t \mathcal{J}$, we apply Lemma 1 , with $X=t^{2} f^{2}(m / t)$ and $Y=T^{2}$. We obtain

$$
\sum_{m \in t \mathcal{J}} P\left(t^{2} f^{2}(m / t)\right)
$$

$$
\begin{aligned}
& =\sum_{m \in t \mathcal{J}}\left(\frac{\sqrt{t}}{\pi} \sqrt{f(m / t)} \sum_{1 \leq n \leq T^{2}} \frac{r(n)}{n^{3 / 4}} \cos (2 \pi \sqrt{n} t f(m / t)-3 \pi / 4)+O\left(T^{\varepsilon}\right)\right) \\
& =\frac{\sqrt{t}}{\pi} \sum_{1 \leq n \leq T^{2}} \frac{r(n)}{n^{3 / 4}}\left\{\sum_{m \in t \mathcal{J}} \sqrt{f(m / t)} \cos (2 \pi \sqrt{n} t f(m / t)-3 \pi / 4)\right\}+O\left(T^{1+\varepsilon}\right) .
\end{aligned}
$$

We shall transform the inner sum here by means of Lemma 2, with

$$
G(\tau):=\sqrt{f(\tau / t)}, \quad F(\tau):=\sqrt{n} t f(\tau / t) .
$$

To do so we put $L:=T$ length $(\mathcal{J})$, and observe that $|\tau-a t| \asymp L$ for all $\tau \in t \mathcal{J}$, where $a$ is the one of $a_{1}, a_{2}$ which is nearer to $\mathcal{J}$. Hence, in view of (3.3),

$$
F^{\prime \prime}(\tau)=\sqrt{n} t^{-1} f^{\prime \prime}(\tau / t) \asymp \sqrt{n} t^{-1}|\tau / t-a|^{\alpha-2} \asymp \sqrt{n} t^{1-\alpha} L^{\alpha-2}
$$

for all $\tau \in t \mathcal{J}\left(\alpha\right.$ the appropriate $\left.\alpha_{i}\right)$, and similarly

$$
F^{(j)}(\tau) \ll \sqrt{n} t^{1-\alpha} L^{\alpha-j} \quad \text { for } j=3,4 .
$$


Furthermore, by (3.4),

$$
G^{(j)}(\tau)=\frac{d^{j}}{d \tau^{j}}(\sqrt{f(\tau / t)}) \ll t^{-j}|\tau / t-a|^{\alpha / 2-j} \ll t^{-\alpha / 2} L^{\alpha / 2-j}
$$

for $\tau \in t \mathcal{J}, j=0,1,2$. We may thus apply Lemma 2 with the parameters

$$
X:=L, \quad Y:=n^{-1 / 2} t^{\alpha-1} L^{2-\alpha}, \quad Z:=(L / t)^{\alpha / 2} .
$$

To simplify the notation in what follows, put

$$
\beta(z):=\sqrt{\frac{f\left(f^{*}(z)\right)}{\left|f^{\prime \prime}\left(f^{*}(z)\right)\right|}} .
$$

After a short computation, Lemma 2 yields

$$
\begin{aligned}
& \sum_{m \in t \mathcal{J}} \sqrt{f(m / t)} \cos (2 \pi \sqrt{n} t f(m / t)-3 \pi / 4) \\
= & \frac{\sqrt{t}}{n^{1 / 4}} \Re\left(-\sum_{k \in \sqrt{n} \mathcal{J}^{*}} \beta(k / \sqrt{n}) e\left(t\left(\sqrt{n} f\left(f^{*}(k / \sqrt{n})\right)-k f^{*}(k / \sqrt{n})\right)\right)\right) \\
& +O\left(n^{-1 / 4} t^{(\alpha-1) / 2} L^{1-\alpha / 2}\right)+O(\log t)
\end{aligned}
$$

where $f^{*}$ denotes the inverse function of $f^{\prime}$ and $\mathcal{J}^{*}$ the image of the closure $\overline{\mathcal{J}}$ of $\mathcal{J}$ under $f^{\prime}$. The contribution of the error terms here to the whole of $(5.2)$ is

$$
\ll t \sum_{1 \leq n \leq T^{2}} \frac{r(n)}{n}+\sqrt{t} \log t \sum_{1 \leq n \leq T^{2}} \frac{r(n)}{n^{3 / 4}} \ll T \log T,
$$

hence small enough. Now $k \in \sqrt{n} \mathcal{J}^{*}$ implies that $f^{*}(k / \sqrt{n}) \in \overline{\mathcal{J}}$. Hence, by (3.3) and the fact that $f^{\prime \prime}$ is bounded away from zero,

$$
\beta(k / \sqrt{n}) \ll L / T \quad \text { for } k \in \sqrt{n} \mathcal{J}^{*} .
$$

Furthermore, by the definition of the tac-function $H$, for all $k \in \mathbb{Z}, n \in \mathbb{Z}^{+}$,

$$
\begin{aligned}
H(-k, \sqrt{n}, 0) & =\max _{(x, y, 0) \in \partial \mathcal{R}}(-k x+\sqrt{n} y)=\max _{a_{1} \leq x \leq a_{2}}(-k x+\sqrt{n} f(x)) \\
& =\sqrt{n} f\left(f^{*}(k / \sqrt{n})\right)-k f^{*}(k / \sqrt{n}) .
\end{aligned}
$$

Hence it will suffice to show that

$$
I(\mathcal{J}, T):=\int_{T}^{2 T} t^{2}|S(\mathcal{J}, t, T)|^{2} d t \ll T^{3+\varepsilon},
$$

where

$$
S(\mathcal{J}, t, T):=\sum_{1 \leq n \leq T^{2}} \frac{r(n)}{n} \sum_{k \in \sqrt{n} \mathcal{J}^{*}} \beta(k / \sqrt{n}) e(t H(-k, \sqrt{n}, 0)) .
$$


Dividing into dyadic subintervals, it is thus enough to show that

$$
\int_{T}^{2 T}\left|\sum_{n \asymp N} \sum_{k \asymp K} \gamma_{n, k} e(t H(-k, \sqrt{n}, 0))\right|^{2} d t \ll T^{1+\varepsilon}
$$

with

$$
\begin{aligned}
& N \ll T^{2}, \quad K \ll \sqrt{N}(L / T)^{\alpha-1}, \\
& \gamma_{n, k}:=\beta(k / \sqrt{n}) \frac{r(n)}{n} \ll L T^{-1+\varepsilon / 2} N^{-1} .
\end{aligned}
$$

Here we used (5.4) and the fact that, in view of (3.3),

$$
\max _{\xi \in \mathcal{J}^{*}}|\xi|=\max _{x \in \overline{\mathcal{J}}}\left|f^{\prime}(x)\right| \ll(L / T)^{\alpha-1} .
$$

By Lemma 5, the left-hand side of (5.6) is

$$
\begin{aligned}
& \ll T^{1+\varepsilon} \frac{L^{2}}{T^{2} N^{2}} N K \\
& \quad \times \max _{H_{0} \ll K+\sqrt{N}} \#\left\{n \asymp N, k \asymp K:\left|H(-k, \sqrt{n}, 0)-H_{0}\right|<1 / T\right\} .
\end{aligned}
$$

According to Lemma 4, this cardinality is

$$
\ll N / T+\sqrt{N}+K \ll \sqrt{N}+K .
$$

To see this, we use the first inequality of $(3.11)$ if $K \ll \sqrt{N}$ and the second one otherwise, with $\delta$ a constant multiple of $\sqrt{N} / K$. Therefore, the left-hand side of (5.6) is overall

$$
\ll T^{1+\varepsilon}(L / T)^{2} \frac{K}{\sqrt{N}}\left(1+\frac{K}{\sqrt{N}}\right) \ll T^{1+\varepsilon}\left((L / T)^{1+\alpha}+(L / T)^{2 \alpha}\right) \ll T^{1+\varepsilon},
$$

with an appeal to (5.7). This completes the proof of (5.6) and thereby, in view of (4.1) and (4.5), that of our Theorem.

6. Concluding remark. We indicate briefly how the pointwise bound (2.3) follows as a by-product from the above analysis. Estimating the righthand side of (5.3) trivially, we get, for $t=T$,

$$
\begin{aligned}
\sum_{m \in t \mathcal{J}} \sqrt{f(m / t)} \cos (2 \pi \sqrt{n} t f(m / t)-3 \pi / 4) \\
\ll n^{1 / 4} t^{1 / 2}\left|\mathcal{J}^{*}\right|+n^{-1 / 4} t^{(\alpha-1) / 2} L^{1-\alpha / 2}+\log t \\
\ll n^{1 / 4} t^{3 / 2-\alpha} L^{\alpha-1}+n^{-1 / 4} t^{(\alpha-1) / 2} L^{1-\alpha / 2}+\log t,
\end{aligned}
$$

in view of (5.7). Using Lemma 1 with

$$
X=t^{2} f^{2}(m / t) \ll t^{2}, \quad Y=L^{2-\alpha} t^{\alpha-1} \ll t,
$$


we obtain, as an obvious variant of (5.2),

$$
\begin{aligned}
\sum_{m \in t \mathcal{J}} P\left(t^{2} f^{2}(m / t)\right) & \\
\ll & \sqrt{t} \sum_{1 \leq n \leq Y} \frac{r(n)}{n^{3 / 4}}\left(n^{1 / 4} t^{3 / 2-\alpha} L^{\alpha-1}+n^{-1 / 4} t^{(\alpha-1) / 2} L^{1-\alpha / 2}+\log t\right) \\
& \quad+L t^{1+\varepsilon} / Y^{1 / 2} \\
\ll & t^{2-\alpha} L^{\alpha-1} Y^{1 / 2}+t^{\alpha / 2} L^{1-\alpha / 2} \log t+t^{1 / 2} Y^{1 / 4} \log t+L t^{1+\varepsilon} / Y^{1 / 2} \\
\ll & L^{\alpha / 2} t^{3 / 2-\alpha / 2+\varepsilon}+L^{1-\alpha / 2} t^{\alpha / 2} \log t+L^{1 / 2-\alpha / 4} t^{\alpha / 4+1 / 4} \log t \\
\ll & t^{3 / 2+\varepsilon} .
\end{aligned}
$$

Acknowledgements. The author is pleased to express his sincere gratitude to an anonymous referee whose extremely valuable comments contributed considerably to clarify and shorten the exposition of this paper.

\section{References}

[1] V. Bentkus and F. Götze, On the lattice point problem for ellipsoids, Acta Arith. 80 (1997), 101-125.

[2] F. Chamizo, Lattice points in bodies of revolution, ibid. 85 (1998), 265-277.

[3] F. Götze, Lattice point problems and values of quadratic forms, Invent. Math. 157 (2004), 195-226.

[4] K. Haberland, Über die Anzahl der Gitterpunkte in konvexen Gebieten, preprint FSU Jena, 1993 (unpublished).

[5] E. Hlawka, Über Integrale auf konvexen Körpern I, Monatsh. Math. 54 (1950), 1-36.

[6] -, Über Integrale auf konvexen Körpern II, ibid., 81-99.

[7] M. N. Huxley, Area, Lattice Points, and Exponential Sums, London Math. Soc. Monogr. 13, Clarendon Press, Oxford, 1996.

[8] A. Iosevich, E. Sawyer, and A. Seeger, Mean square discrepancy bounds for the number of lattice points in large convex bodies, J. Anal. Math. 87 (2002), 209-230.

[9] A. Ivić, The Laplace transform of the square in the circle and divisor problems, Studia Sci. Math. Hungar. 32 (1996), 181-205.

[10] A. Ivić, E. Krätzel, M. Kühleitner, and W. G. Nowak, Lattice points in large regions and related arithmetic functions: Recent developments in a very classic topic, in: Proc. Conf. on Elementary and Analytic Number Theory ELAZ'04 (Mainz, 2006), W. Schwarz and J. Steuding (eds.), Franz Steiner Verlag, 2006, 89-128.

[11] H. Iwaniec and E. Kowalski, Analytic Number Theory, Colloq. Publ. 53, Amer. Math. Soc., Providence, RI, 2004.

[12] V. Jarník, Über die Mittelwertsätze der Gitterpunktlehre, V. Abh., Čas. Mat. Fys. 69 (1940), 148-174.

[13] D. G. Kendall, On the number of lattice points inside a random oval, Quart. J. Math. (Oxford) 19 (1948), 1-26.

[14] E. Krätzel, Lattice Points, Deutscher Verlag Wiss., Berlin, 1988. 
[15] E. Krätzel, Analytische Funktionen in der Zahlentheorie, Teubner, Stuttgart, 2000.

[16] - Lattice points in three-dimensional large convex bodies, Math. Nachr. 212 (2000), $77-90$.

[17] —, Lattice points in three-dimensional convex bodies with points of Gaussian curvature zero at the boundary, Monatsh. Math. 137 (2002), 197-211.

[18] - Lattice points in some special three-dimensional convex bodies with points of Gaussian curvature zero at the boundary, Comment. Math. Univ. Carolin. 43 (2002), $755-771$.

[19] E. Krätzel und W. G. Nowak, Eine explizite Abschätzung für die Gitter-Diskrepanz von Rotationsellipsoiden, Monatsh. Math., to appear.

[20] M. Kühleitner, Lattice points in bodies of revolution in $\mathbb{R}^{3}$ : an $\Omega_{- \text {-estimate for the }}$ error term, Arch. Math. (Basel) 74 (2000), 234-240.

[21] M. Kühleitner and W. G. Nowak, The lattice point discrepancy of a body of revolution: Improving the lower bound by Soundararajan's method, ibid. 83 (2004), 208-216.

[22] Y.-K. Lau, On the mean square formula of the error term for a class of arithmetical functions, Monatsh. Math. 128 (1999), 111-129.

[23] W. Müller, On the average order of the lattice rest of a convex body, Acta Arith. 80 (1997), 89-100.

[24] -, Lattice points in large convex bodies, Monatsh. Math. 128 (1999), 315-330.

[25] W. G. Nowak, On the average order of the lattice rest of a convex planar domain, Proc. Cambridge Philos. Soc. 98 (1985), 1-4.

[26] - On the mean lattice point discrepancy of a convex disc, Arch. Math. (Basel) 78 (2002), 241-248.

[27] M. Peter, Lattice points in convex bodies with planar points on the boundary, Monatsh. Math. 135 (2002), 37-57.

[28] H. Rademacher, Topics in Analytic Number Theory, Springer, Berlin, 1973.

Institute of Mathematics

Department of Integrative Biology

Universität für Bodenkultur Wien

Gregor Mendel-Straße 33

A-1180 Wien, Austria

E-mail: nowak@boku.ac.at

Received on 12.6.2006

and in revised form on 27.11.2006 